\section{Tubulin site interpretation}

SiR-Paschal et al. ${ }^{1}$ conclude that an influence both tubulin assembly and the amino-acid sequence at the carboxy binding of MAP2 and dynein, raising the terminus of $\alpha$ - and $\beta$-tubulin is a common possibility that some MAPs interact SEQUENCES AND OCCURRENCE OF $\beta$-TUBULIN.

\begin{tabular}{llccc}
\hline Class & Sequence from residue 422 & Brain & $\begin{array}{c}\text { Red blood } \\
\text { cells }\end{array}$ \\
I & yqqyqdat aEEEED-FGEEAKEEA & & 10 \\
II & yqqyqda t aDEQGE-FEEEGEEDEA & 58 & 0 \\
III & yqqyqdat aEEEGEMYEDDEEESEQGAK & 25 & 0 \\
IV & yqqyqdataEEEGE-FEEEAEEEAE & 13 & 10 \\
V & yqqyqEat aNDGEEAFEDDEEEINE & 0 & 0 \\
VI & yqqyqdataDVEE--YEEAEASPEKET & 0 & 80
\end{tabular}

Isotype classes are shown to the left of the amino acid sequences. The last two columns show the percentages of total brain and erythrocyte tubulin, represented by $\beta$, of the classes shown (our unpublished observations).

site of interaction for the microtubuleassociated proteins (MAPS) cytoplasmic dynein and MAP2. The identity of the site was inferred from the failure of tubulin subunits, cleaved with proteases near their carboxy termini at sites mapped by specific tubulin antibodies, to interact productively with dynein or MAP2. The conclusion is appealing because it suggests a common, perhaps universal, MAPbinding motif $\mathrm{E}(\mathrm{G} / \mathrm{A}) \mathrm{EE}$.

But we believe this interpretation requires fuller consideration. Although the authors note that the same binding domain is found on known $\alpha$ - and $\beta$ tubulins from various species, a comparison of the subunits that would compete for MAP binding within a single organism, or even within the same cell, is also relevant. The table shows the carboxy-terminal sequences of all the $\beta$-tubulins in the chicken $^{2}$. Remarkably, the supposed MAP-binding domain does not lie in a domain conserved among all $\beta$-isotypes, but in the hypervariable region that distinguishes the isotype classes from each other ${ }^{3}$. Only three of the six subunits carry the supposed binding sequence which, indeed, is missing in Class III $\beta$-tubulin expressed, like MAP2, exclusively in neurons.

It is also relevant that there are several high-affinity binding sites $\left(K_{\mathrm{d}}=5 \mu \mathrm{M}\right)$ on MAP2 (and also on tau) for a peptide containing residues $422-434$, (ref. 4) which is almost invariant among known $\beta$-tubulin.

Finally, one of us (D.B.M.) has shown that MAP2 binds nearly equivalently (and with high affinity, $K_{\mathrm{d}}=0.1 \mu \mathrm{M}$ ) to microtubules composed either of brain or erythrocyte tubulin, even though the class VI $\beta$-tubulin mostly involved does not contain the Paschal sequence and is very different from the other isotypes.

Thus the primary site of MAP interaction probably lies not where Paschal et al. ${ }^{1}$ suggest, but in the neighbouring conserved region of $\beta$-tubulin molecules. Yet the acidic variable carboxy termini clearly motility even in neurons in which MAP2 is abundant. specific tubulin isotypes. Selective binding to $\beta$ tubulin isotypes specific to the brain could be significant in relation to the observation of Paschal et al. that teraction of dyenin with tubulin in the brain: preferential MAP binding to particular isotypes could HARISH C. JOSHI

Department of Biological Chemistry, DOUGLAS B. MURPHY

Department of Cell Biology,

School of Medicine,

Johns Hopkins University,

Baltimore, Maryland 21205 , USA

Paschal et al. RePly - Murphy et al., in discussing the importance of the tubulin sequence identified in our study, fail to address the role of $\alpha$-tubulin in the interaction with dynein and MAP2, and ignore evidence contradicting a role for the more highly conserved $\beta$-tubulin region.

Our results ${ }^{1}$ addressed $\alpha$-tubulin most directly. In chicken blood-forming tissue and circulating erythrocytes the predominant $\alpha$-tubulin isoform is $\alpha-1$, which, in fact, contains the sequence $\mathrm{EGEE}^{5,6}$. About 20 per cent of chicken erythrocyte $\beta$-tubulin also contains the related sequence EAEE (see table). In view of the well-known substoichiometric binding of MAPs to microtubules at saturating MAP concentrations, there is a substantial level of $\alpha$ - and $\beta$-tubulin isoforms in the erythrocyte containing our proposed binding sequence. We also note that the chief $\beta$-tubulin in the erythrocyte contains the sequence EEAEA, which could be functionally related to our proposed EEAEE.

With respect to earlier evidence on the MAP2-tubulin interaction, Maccioni

1. Paschal, B. M. et al. Nature 342, 569-572 (1989)

2. Monteiro, M. J. \& Cleveland, D. W. J. molec. biol. 199. 439-446 (1988)

3. Sullivan, K. F. \& Cleveland, D. W. Proc. natn. Acad. Sci. U.S.A. 83, $4327-4331$ (1986).

4. Maccioni, R. et al. EMBO J. 7, 1957-1963 (1988)

5. Pratt, L. F. \& Cleveland, D. W. EMBO J. 7. 931-940 (1988)

6. Murphy, D. B. \& Wallis, K. T. J. biol. Chem. 258, $7870-$ 7875 (1983)

7. Littauer, U. Z. et al. Proc. natn. Acad. Sci. U.S.A. 83 $7162-7166$ (1986) preferentially with MAP2 blocks the inDon W. CleVELAND et $a l .{ }^{4}$ reported on the affinity of the $\beta$-tubulin peptide $422-434$ but made no direct test of the extreme carboxy-terminal region. Littauer et al. ${ }^{7}$ found MAP2 binding to peptides from several regions in $\alpha$ - and $\beta$-tubulin but, significantly, no binding to the highly conserved $\beta$ tubulin sequence consisting of amino acids 416-431.

BRYCE M. PASCHAL

ROBERT A. OBAR

RICHARD B. VALLEE

Cell Biology Group,

Worcester Foundation for Experimental Biology,

Shrewsbury,

Massachusetts 01545 ,

USA

\section{Sex, growth and chance}

SIR-In his News and Views article ${ }^{1}$, Paul Burgoyne suggested that the $Z F Y$ and $Z F X$ zinc-finger genes on the human sex chromosomes may speed up the development of somatic cells as a prerequisite for the differentiation of the testis. Lack of $Z F Y$ could therefore lead to failure of masculinization in XX patients presumed to carry the testis-determining $T D Y$ gene. Phenotypically, such patients can be male, female or hermaphrodite, but neither of the explanations discussed by Burgoyne to account for this variability $-\mathrm{X}$ inactivation and functional interchangeability of $Z F Y$ and $Z F X$ gene products with quantitatively different effects - is supported by evidence from human cytogenetics.

When trying to explain the genetics of sex reversal, we need to abandon the search for models which have been successful in unravelling the basis of enzyme defects and confront the problem of cells growing at different rates, where chance may have the last word ${ }^{2}$. When I first suggested that sex differentiation is initiated by differential growth ${ }^{3}$, I also suggested that threshold dichotomy ${ }^{4}$, or quasicontinuous variation $^{5}$ provided the underlying mechanism. Here, a genetic system resulting in an apparently qualitative difference is caused by quantitative variation with a threshold effect, giving rise to two (almost) distinct classes. The concept was originally invoked to explain the basis of characters like extra toes in guinea pigs ${ }^{4}$ and missing teeth in mice ${ }^{5}$, which have an obvious genetic basis but are not transmitted according to mendelian rules. Neuraltube defects in mice can be caused by incompatible growth rates between different interacting tissues ${ }^{6}$, providing further support for the idea that the genetic basis of sex reversal is similar to that of spina bifida, which also has a genetic basis without a clear-cut pattern of inheritance. 